\title{
Optical Properties of Tensile-strained Barrier GaAsP/GaAs Intermixed Quantum Well Structure
}

\author{
Steven K. H. Sim, Michael C.Y. Chan and E. Herbert Li, Senior Member, IEEE \\ University of Hong Kong, Department of Electrical and Electronic Engineering, \\ Pokfulam Road, Hong Kong.
}

\begin{abstract}
The tensile-strained barrier GaAsP/GaAs quantum well (QW) structures fabricated on GaAs substrate has a remarkable potential for novel properties of laser structures. In this structure, the GaAs QW layer is embedded with tensile-strained GaAsP barriers grown on GaAs substrates. Recent research has reported that the threshold current densities with tensilestrained barrier QW laser are comparable with GaAsP/AlGaAs tensile-strained well QWs. In the case of tensile-strained barrier GaAsP/GaAs QW, a small amount of light-hole $(\mathrm{LH})$ and heavy-hole $(\mathrm{HH})$ splitting is attainable within a large range of well width and $\mathrm{P}$ compositions. By a suitable choice of material and structure parameters, it is possible to cause the coincidence of energy levels of $\mathrm{HH}$ and $\mathrm{LH}$ resulting in polarization-independent operation devices. In order to shift the $\mathrm{HH}$ and LH energy levels, the concept of band-gap engineering is a useful tool to accept the particular devices operation. Basically, intermixing process is one of easy ways to achieve the modification of bandstructure. During the process the asgrown square-QW compositional profile is modified to a graded profile thereby altering the confinement profile and subband structure in the QW. In this paper, the optical properties of intermixed GaAs QW with tensile-strained GaAsP barriers are reported.
\end{abstract}

Keywords: III-V Semiconductor, Quantum Well, GaAsP/GaAs, Tensile-strained barrier, Band-gap engineering, Interdiffusion, Polarization-independent, Optical gain

\section{INTRODUCTION}

Modification of band structures to improve the performance and characteristics of optical devices has been one of the major research area for band structure engineering. For quantum-well (QW) devices, combined both the factors of quantum size and strain induced in layered structure allowed a higher flexibility for band structure engineering. This can improve are certain characteristics of laser diode, including higher differential gain, lower threshold current, higher output power, enhanced linewidth and modulation bandwidth. ${ }^{1-6 .}$ Both the compressive and tensile strain has an important impact on the band structure engineering, while most of the early works were focus on the effect of compressive-strained quantum-well. At 1992 the tensile-strained quantum-well diode laser has reported with lower current densities, and high temperature operation, and polarization insensitive devices utilizing these structures. ${ }^{7-9}$.

In unstrained quantum-well case, the heavy-hole state is the highest valence band state due to the result of the quantum effect alone, and the depth and width of the wells varies the energy difference between the light-hole and heavy-hole bands. The lowest evergy transition which dominates in the optical spectra of such structures is the conduction band to the closest valence band state with unequal oscillator strengths for TE and TM polarizations. In case of compressive-strain quantumwell, the bandgap will be increase and the compressive-strain effect will separate the light-hole and heavy-hole further apart, which is similar to the effect of quantum confinement. While the tensile-strain in barrier decrease the bandgap and the light-hole is being lifted above the heavy-hole, which is opposite to the quantum confinement effect. This result show that the hole states can be vary with correct quantum size and carefully designed strain effect.

The relationship between the bandgap energy and lattice constant is inversely proportional, the well layer must be made very thin in order to achieve the two hole merge together. Heterostructure of GaAs well, GaAsP barrier growth on GaAs substrate provide a tensile-strained barrier structure without involving complicated quaternary alloys, and it has been studied widely. The $\mathrm{P}$ concentration in barrier and the well-width is the two element that can be vary to tune for a particular band structure. One of the structures that researches have great interest on is the merging of the light-hole and the heavy-hole band. The merge of the two band would enhance electroabsorption, and polarization independence, polarization independence offer advantage to various device application. When relating the $\mathrm{P}$ concentration and the well width in order 
to merge the two band, their relationship is inversely proportional. Which mean a thinner well width is required as the $\mathrm{P}$ concentration increase. When $\mathrm{P}$ concentration increase the optical gain is also enhance, while the band gain become wider due to the narrow well effect.

The post-growth process of thermally induced composition intermixing of QWs has becoming a popular technique for material tuning and which, is proposed here. The band structure ${ }^{10}$ and types of induced strain ${ }^{11}$ intermixing process changes in the interdiffused QW (DFQW). Consequently, joint density of states, optical transition element and other optical parameters will change accordingly. The interdiffusion has been studied in various material systems. This includes the more popular $A l G a A s / G a A s^{10-12}, \operatorname{InGaAs} / G a A s^{13-14}, \operatorname{InGaAs} / \operatorname{In} P^{15-16}$, and $\mathrm{Si} / G e^{17}$. There are also work reported in the not so common systems, such as $G a A s S b / G a A s^{18}$ and $C d S e / Z n S e^{19}$. The interdiffusion techniques are not only applicable in the tunability of device, enhancement in operation can also be obtained. In order to modify the device performance and to achieve tunability, a well-controlled interdiffusion technique is essential. By using impurity-induced enhanced intermixing, through ion implantation $^{20}$ or diffusion ${ }^{21}$, and alternatively by impurity-free vacancy enhanced intermixing technique ${ }^{22}$ through a masking process, selective area intermixing can be achieved planarly. These techniques not only have provided the wavelength tunability of devices ${ }^{23}$, but it can also improve device performance ${ }^{24}$ and are promising technologies for the integration of photonics IC's ${ }^{25}$.

We demonstrated the interdiffusion technique to tune the band structure. The interdiffusion technique is a thermal process consist of consituant atom moving across the hetero-interface. This will change both the band structure and its optical properties of a quantum-well. Interdiffusion technique shows its ability to merge both the light-hole and heavy-hole band while maintaine a reasonable optical gain. One of the major advantage of using interdiffusion technique for band structure tunning is because its accuracy is much higher than for growing an precise as-grown heterostructure. Themal annealing allow a more precise tunning and the optical gain is enhanced with small amount of diffusion into the structure. This improve some of the major factors in application to quantum-well laser diode.

In this paper, we present a detailed theoretical analysis of the group-V interdiffused GaAsP/GaAs tensile-strain barrier QWs. To calculate the electron and hole wave function in QW, the multi-band effective mass theory is used: A parabolic band model and $4 \times 4$ Luttinger-Kohn Hamiltonian with the strain-component in a block diagonal, or called as the Pikus-bir Hamiltonian are used for the conduction and valence bands respectively. After the subband structures are obtained, we can calculated the optical gain using the Fermi golden rule. The optical gain spectra are calculated by the density matrix approach. This includes the confinement profiles, LH-HH splitting and optical gain. In section II, we will present the models for group-V interdiffusion, induced strain, band structure and optical properties. In section III, the as-grown structure are selected to demonstrate how the $\mathrm{LH}-\mathrm{HH}$ splitting and optical gain are affected by the technique of intermixing. Finally the conclusions are drawn.

\section{MODELLING}

\subsection{Effects of disordering}

In our diffusion model, it is assumed that Fick's second law is obeyed in the QW layers and all atoms movement through the interface between the adjacent hetro-layers have the same diffusion coefficient. Interdiffusion across the heterointerface alters the composition profile across the QW structure. In GaAsP/GaAs QW structures only the interdiffusion of group-V atoms occurs, i.e. As and $\mathrm{P}$ atoms, since there is no $\mathrm{Ga}$ concentration gradient across the interface. The diffusion of group $\mathrm{V}$ atoms in the quantum well structure is usually described by the Fick's law with constant diffusion coefficients in both the well and barrier layers. The composition profile after interdiffusion is characterized by a diffusion length $L_{d}$, which is defined as $L_{d}=\sqrt{ }(D t)$, where $D$ is the diffusion coefficient and $t$ is the annealing time of thermal processing. Consider a single $\mathrm{GaAsP} / \mathrm{GaAs} \mathrm{QW}$ with the as-grown $\mathrm{P}$ mole fraction equal to $\mathrm{x}_{0}$, the compositional profile of $\mathrm{P}$ after interdiffusion is given by:

$$
x(z)=x_{0}\left\{1-\frac{1}{2}\left[\operatorname{erf}\left(\frac{L_{z}+2 z}{4 L_{d}}\right)+\operatorname{erf}\left(\frac{L_{z}-2 z}{4 L_{d}}\right)\right]\right\}
$$

where $\mathrm{z}$ denotes the coordinate along the crystal growth direction and $\mathrm{L}_{\mathrm{z}}$ is the as-grown well width and the $\mathrm{QW}$ is centered at $\mathrm{z}=0$. 


\subsection{Strain}

Lattice mismatch between thin well and thick barrier QW materials can be taken up by strain and results in a pseudomorphic QW such that an uniform lattice constant can be found through out the whole structure. This tetragonal deformation results in strain and which is, perpendicular to the hetero-interface. Assuming that the growth direction $\mathrm{z}$ is along $\langle 001\rangle$, the $G a A s P$ well layer is subjected to a biaxial tensile in-plane strain parallel to $\mathrm{x}$ along $\langle 100\rangle$ and to $\mathrm{y}$ along $\langle 010\rangle$, and a uniaxial shear strain parallel to $\mathrm{z}$ along $<001>$. The in-plane strain across the well will vary according to the composition of alloy concentration after interdiffusion. Hence the biaxial in-plane strain and uniaxial shear strain after interdiffusion, are given by :

$$
\begin{gathered}
\varepsilon_{x x}=\varepsilon_{y y}=\varepsilon(x) \\
\varepsilon_{z z}=-2\left[c_{12}(x) / c_{11}(x)\right] \varepsilon(x) \\
\varepsilon_{x y}=\varepsilon_{y z}=\varepsilon_{z x}=0
\end{gathered}
$$

where $\varepsilon(\mathrm{x})$ is misfit factor between the well and the barrier such that it defines to be negative for compressive strain, and $\mathrm{c}_{\mathrm{ij}}$ $(\mathrm{x})$, are the elastic stiffness constants. The change in the bulk bandgap, $\mathrm{S}_{\perp}(\mathrm{x})$, due to the hydrostatic component of strain is given by:

$$
S_{1}(x)=-2 a(x)\left[1-c_{12}(x) / c_{11}(x)\right] \varepsilon(x),
$$

where $\mathrm{a}(\mathrm{x})$ is the hydrostatic deformation potential calculated from:

$$
\mathrm{a}(\mathrm{x})=-\frac{1}{3}\left[\mathrm{c}_{11}(\mathrm{x})+2 \mathrm{c}_{12}(\mathrm{x})\right] \frac{d E_{g}(x)}{d P}
$$

where $\mathrm{dE}_{\mathrm{g}} / \mathrm{dP}$ is the hydrostatic pressure coefficient of the lowest direct energy gap $\mathrm{E}_{\mathrm{g}}$. The splitting energy, $\mathrm{S}_{/ /}(\mathrm{x})$, between the HH and LH band edges induced by the shear component of strain is given by :

$$
\mathrm{S}_{/ /}(\mathrm{x})=-\mathrm{b}(\mathrm{x})\left[1+2 \mathrm{c}_{12}(\mathrm{x}) / \mathrm{c}_{11}(\mathrm{x})\right] \varepsilon(\mathrm{x})
$$

where $b(x)$ is the shear deformation potential. The coupling between the LH and split-off band gives rise to asymmetric heavy-hole to light hole splitting, so that

$$
\begin{gathered}
S_{/ H H}(x)=S_{/ /}(x) \\
S_{/ / L H}(x)=-1 / 2\left[S_{/ /}(x)+\Delta_{0}(x)\right]+1 / 2\left[9\left\{S_{/ /}(x)\right\}^{2}+\left\{\Delta_{0}(x)\right\}^{2}-2 S_{/ /}(x) \Delta_{0}(x)\right]^{1 / 2}
\end{gathered}
$$

where $\Delta_{\mathrm{o}}(\mathrm{x})$ is the spin-orbit splitting.

The QW confinement potential after the intermixing process, $\mathrm{U}_{\mathrm{r}}(\mathrm{x})$, is obtained by modifying the unstrained potential profile after processing, $\Delta \mathrm{E}_{\mathrm{r}}(\mathrm{x})$, by the variable strain effects, and is given by:

$$
\mathrm{U}_{\mathrm{r}}(\mathrm{x})=\Delta \mathrm{E}_{\mathrm{r}}(\mathrm{x})-\mathrm{S}_{\perp \mathrm{r}}(\mathrm{x}) \pm \mathrm{S}_{|| r}(\mathrm{x})
$$

where $S_{\perp r}(x)=Q_{r} S_{\perp}(x)$, the ' + ' and '-' signs represent the confined HH and LH profiles, respectively, and $S_{\| c}(x)=0$.

\subsection{Band Structure}

To calculate the electron and hole wave function in QW, the multiband effective mass theory is applied. For most III-V semiconductors such as $G a A s$-based materials, it is a good approximation that the conduction and valence bands are decoupled. A parabolic bands model and Luttinger-Kohn Hamiltonian with strain components are used for the conduction and valence bands respectively. The electron states near the conduction subband edge are assumed to be almost purely slike and nondegenerate (excluding spin), while the hole states near the valence subband edge are almost purely p-like and 
four-fold degenerate (including spin). The envelope function scheme is adopted to describe the slowly varying (spatially extended) part of the wavefunction.

The wavefunctions of the electron and hole subband edge at the zone centre of $\Gamma_{6}$ valley symmetry can be calculated separately, using the Ben-Daniel and Duke model ${ }^{26}$ by the one-dimensional Schrodinger-like equation, which is written as follows:

$$
-\frac{\hbar^{2}}{2} \frac{\mathrm{d}}{\mathrm{dz}}\left[\frac{1}{\mathrm{~m}_{\mathrm{r}}^{*}(\mathrm{z})} \frac{\mathrm{d} \psi_{\mathrm{r} \ell}(\mathrm{z})}{\mathrm{dz}}\right]+\mathrm{U}_{\mathrm{r}}(\mathrm{z}) \cdot \psi_{\mathrm{r} \ell}(\mathrm{z})=\mathrm{E}_{\mathrm{r} \ell} \psi_{\mathrm{r} \ell}(\mathrm{z})
$$

where $\psi_{\mathrm{r} 1}(\mathrm{z})$ is the wavefunction of the $1^{\text {th }}$ subband for electrons $(\mathrm{r}=\mathrm{cl})$ or holes $(\mathrm{r}=\mathrm{v} 1)$, respectively; $\mathrm{m}_{\mathrm{r}}^{*}(\mathrm{z})$ is the corresponding carrier effective mass in the $\mathrm{z}$ direction; $\mathrm{E}_{\mathrm{r} 1}$ is the subband-edge energy. Equation (9) is solved numerically using a finite difference method with the above confinement profile.

For valence band structure, it is necessary to diagonalize the Luttinger-Kohn Hamiltonian with appropriate confinement potentials for heavy and light hole. The envelope function at finite $\mathrm{k}_{/ /}$depend on $\mathrm{k}_{/ /}$as a result of the mixing of the heavy and light hole bands. In this paper, the effective Hamiltonian approach described in $\mathrm{Chan}^{27}$ to calculate the valence subband structure is used. In this approach, the hole envelope function $\psi_{\mathrm{v} 1}\left(\mathrm{k}_{/ /}, \mathrm{z}\right)$ at any finite $\mathrm{k}_{/ /}$not too far away from the zone centre $\left(\mathrm{k}_{/ /} \neq 0\right)$ can be expressed as a linear combination of the envelope functions $\psi_{\mathrm{v} 1}(\mathrm{z})$ at $\mathrm{k}_{/ /}=0$ as follows

$$
\psi\left(\mathrm{k}_{\|}, \mathrm{z}\right)=\sum_{\ell=1}^{\mathrm{N}} \sum_{\mathrm{v}=-3 / 2}^{3 / 2} \mathrm{~d}_{\mathrm{v}, \ell}\left(\mathrm{k}_{\|}\right) \psi_{\mathrm{V}, \ell}(\mathrm{z})
$$

where $\psi_{\mathrm{v} 1}(\mathrm{z})$ are obtained by solving the equation (9). This approximation is accurate within a limited range of $\mathrm{k}_{/ /}$, as only a finite basis set is used in the linear expansion. A basis set of about 40 envelop functions is used in the present calculation and the results obtained are accurate within the operation energy range of QW laser. The effective Hamiltonian obtained is

$$
\left(\begin{array}{cccc}
\mathrm{E}_{3 / 2}+\mathrm{S}_{/ / \mathrm{HH}} & \mathrm{C} & \mathrm{B} & 0 \\
\mathrm{C}^{*} & \mathrm{E}_{-1 / 2}-\mathrm{S}_{/ / \mathrm{LH}} & 0 & \mathrm{~B}^{\mathrm{T}} \\
\mathrm{B}^{*} & 0 & \mathrm{E}_{+1 / 2}-\mathrm{S}_{/ / \mathrm{LH}} & \mathrm{C}^{\mathrm{T}} \\
0 & \mathrm{~B}^{*} & \mathrm{C}^{*} & \mathrm{E}_{-3 / 2}+\mathrm{S}_{/ / \mathrm{HH}}
\end{array}\right)
$$

$$
\begin{aligned}
& \text { where } \mathrm{C}_{\ell \ell^{\prime}}=\left(\frac{3}{4}\right)^{\frac{1}{2}} \frac{\hbar^{2}}{\mathrm{~m}_{\mathrm{o}}} \gamma_{2}\left(\mathrm{k}_{\mathrm{x}}-\mathrm{ik} \mathrm{k}_{\mathrm{y}}\right){ }^{2} \int_{0}^{\mathrm{L} f} \mathrm{dz} \psi_{-3 / 2, \ell}(\mathrm{z}) \psi_{1 / 2, \ell^{\prime}}(\mathrm{z}) \\
& \mathrm{B}_{\ell \ell^{\prime}}=(3) \frac{1}{2} \frac{\hbar^{2}}{\mathrm{~m}_{\mathrm{o}}} \gamma_{2}\left(-\mathrm{k}_{\mathrm{x}}-\mathrm{ik}_{\mathrm{y}}\right)^{2} \int_{0}^{\mathrm{L}_{\mathrm{f}}} \mathrm{dz} \psi_{3 / 2, \ell}(\mathrm{z}) \frac{\partial}{\partial \mathrm{z}} \psi_{-1 / 2, \ell^{\prime}}(\mathrm{z}) \\
& \mathrm{E}_{ \pm 3 / 2, \mathrm{ss}}=\delta_{\mathrm{ss}} \cdot \mathrm{E}_{\mathrm{Hs}}-\frac{\hbar^{2}}{2 \mathrm{~m}_{\| \mathrm{H}}} \mathrm{k}_{\|}^{2} \\
& \mathrm{E}_{ \pm 1 / 2, \mathrm{ss}}=\delta_{s s^{\prime}}, \mathrm{E}_{\mathrm{Ls}}-\frac{\hbar^{2}}{2 \mathrm{~m}_{\| \mathrm{L}}} \mathrm{k}_{\|}^{2}
\end{aligned}
$$

where $\gamma_{2}$ is the Luttinger parameter.

\subsection{Optical Gain}

The propagation direction of the generated photon is parallel to the QW layers, and the gain spectrum is calculated by the density matrix approach. The gain spectrum due to the transition between the conduction subband $p$ and the valence subband $\mathrm{q}$ is given by 


$$
g_{p q}(E)=\frac{2 \pi q^{2} \hbar}{(2 \pi)^{2} n \varepsilon_{0} c m_{0}^{2} L_{w} E} \cdot \int d k\left|\hat{e} \cdot P_{p q}\left(k_{/ /}\right)\right|^{2} \rho\left(E_{p}^{e}\left(k_{/ /}\right)-E_{q}^{h}\left(k_{/ /}\right)-E\right) \times\left[f^{e}\left(E_{p}^{e}\left(k_{/ /}\right)\right)-f^{h}\left(E_{q}^{h}\left(k_{/ \prime}\right)\right)\right]
$$

where $q$ is the electric charge, $\mathrm{n}$ is the refractive index, $\varepsilon_{0}$ is the dielectric constant of the vacuum, $\mathrm{c}$ is the speed of light, $\mathrm{L}_{2}$ is the width of the quantum well, $\mathrm{E}$ is the photon energy, $\mathrm{P}_{\mathrm{pq}}$ is the optical matrix element, $\hat{e}$ is a unit vector along the polarization direction of the optical electric field, and $\mathrm{f}^{\mathrm{e}}$ and $\mathrm{f}^{\mathrm{h}}$ are the Fermi distribution function for electron and hole in the conduction and valence subband, respectively. The spectral broadening of each transition is included, and the optical gain in a single quantum well structure is obtained with some spectral lineshape function over all transition energies $E^{\prime}$. L(E-E') is a Lorentzian line-broadening function and is given by

$$
L\left(E-E^{\prime}\right)=\frac{1}{\pi} \frac{\hbar / \tau_{\text {in }}}{\left(E^{\prime}-E\right)^{2}+\left(\hbar / \tau_{\text {in }}\right)^{2}}
$$

where $\tau_{\mathrm{in}}$ is the intraband relaxation time.

\section{RESULTS AND DISCUSSION}

The as-grown structure analyzed here is an $G a A s_{l-x} P_{x} / G a A s$ single quantum well, with an as-grown well width $\mathrm{L}_{z}=30,60$, and $100 \AA$. The as-grown QW is assumed to be fabricated on GaAs substrate with $\mathrm{GaAs}$ in the well and GaAsP in the barrier forming a tensile-strain barrier $\mathrm{QW}$ structure. The results for various diffusion lengths and $\mathrm{P}$ concentrations are obtained. In our calculation, the as-grown $\mathrm{P}$ concentration in the quantum well $\left(\mathrm{x}_{\mathrm{o}}\right)$ is set to be $0.05,0.2$ and 0.4 so as to create different degrees of tensile strains. All the parameters were determined by Vegard's Law between the binary parameters at room temperature. The conduction band offset $\mathrm{Q}_{c}$ of $\mathrm{GaAsP} / \mathrm{GaAs}$ heterostructures, $\mathrm{Q}_{c}=0.57$, was used. The parameters of binary compounds used in calculation are listed in Table I. For each $\mathrm{P}$ concentration case, the group-V interdiffusion will be considered as represented by their respective diffusion lengths $\left(L_{d}\right)$. Examples of the interdiffusion profiles of group $-\mathrm{V}$ interdiffusion are shown in Fig.1, with which an error-functional profile is used in the diffusion process based on Fick's law

Table I. Material parameters of GaAs and $\mathrm{GaP}$ used in the numerical calculations.

\begin{tabular}{|l|l|l|l|}
\hline & GaAs & GaP & Units \\
\hline $\mathrm{a}_{0}$ & 5.6533 & 5.4505 & $\AA$ \\
\hline $\mathrm{c}_{11}$ & 11.9 & 14.05 & $\times 10^{11} \mathrm{dyn} / \mathrm{cm}^{2}$ \\
\hline $\mathrm{c}_{12}$ & 5.38 & 6.203 & $\times 10^{11} \mathrm{dyn} / \mathrm{cm}^{2}$ \\
\hline $\mathrm{dE}_{\mathrm{g}} / \mathrm{dP}$ & 11.3 & 10.7 & $\times 10^{-6} \mathrm{eV} / \mathrm{bar}$ \\
\hline $\mathrm{b}$ & -1.7 & -1.5 & $\mathrm{EV}$ \\
\hline $\mathrm{m}_{\mathrm{c}}$ & 0.068 & 0.33 & $\mathrm{~m}_{0}$ \\
\hline $\mathrm{m}_{\mathrm{HH}}$ & 0.5 & 0.54 & $\mathrm{~m}_{0}$ \\
\hline $\mathrm{m}_{\mathrm{LH}}$ & 0.068 & 0.16 & $\mathrm{~m}_{0}$ \\
\hline $\mathrm{Eg}\left(\mathrm{GaAs}_{1-\mathrm{x}} \mathrm{P}_{\mathrm{x}}\right)$ & $1.424+1.150 \mathrm{x}+0.176 \mathrm{x}^{2} \quad(\mathrm{x}<0.45)$ & $\mathrm{eV}$ \\
\hline
\end{tabular}

For an as-grown $\mathrm{P}$ concentration set to be 0.2 , there is an $0.72 \%$ tensile strain produced in the barrier. As the interdiffusion proceeds, compositional disordering changes the tensile strain accordingly. The tensile strain will also induce in the well. At $L_{d}=20 \AA$, the tensile strain will be $0.2 \%$ in the well centre, as shown in Fig. 2 . When the concentration of P are chosen to be 0.05 and 0.4 which an $0.18 \%$ and $1.43 \%$ tensile strain are found in as-grown barriers respectively. The variation of strain has similar trend as those in $\mathrm{GaAs}_{0.8} \mathrm{P}_{0.2}$.

The valence band confinement potentials of interdiffused quantum wells with phosphorous $\mathrm{x}_{\mathrm{o}}$ equal to 0.2 are plotted in Fig. 3 for heavy and light holes. Owing to the lack of space, results for holes, $x_{0}=0.05$ and 0.4 are not shown as the main features are very similar to those in Fig.3. In the interdiffusion, the phosphorous concentration in the well increases with $\mathrm{L}_{\mathrm{d}}$ as $A s$ atoms diffuse into the barriers from the well. The confinement potentials have an error function profile as well as the distribution of $P$ can be described by the error functions. The band structure can be modified by interdiffusion. They are: 


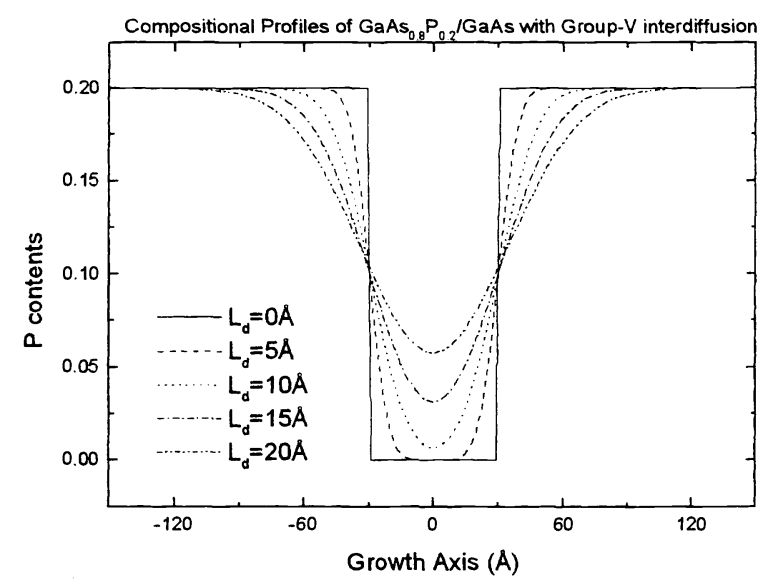

Fig.1 The $\mathrm{P}$ compositional profiles of $\mathrm{GaAs}_{0.8} \mathrm{P}_{0.2} / \mathrm{GaAs} \mathrm{QW}$ with as-grown well width of $60 \AA$ for various diffusion length

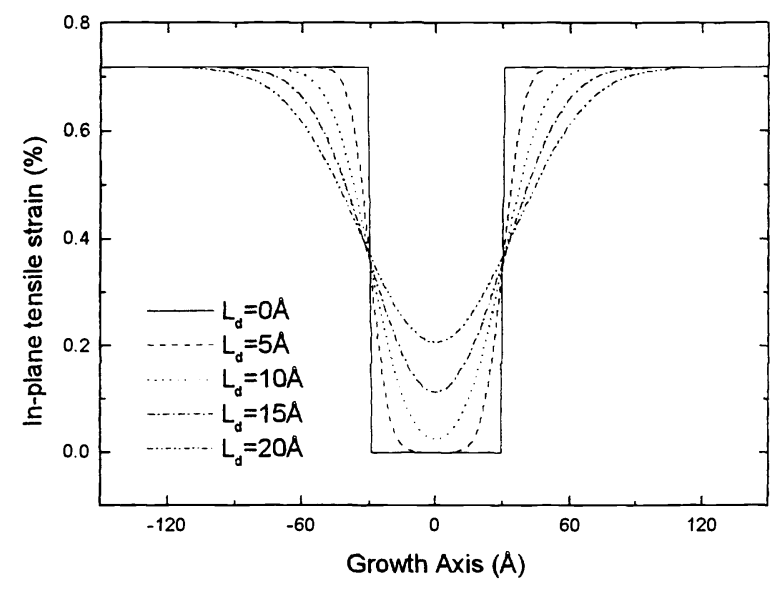

Fig.2 In-plane strain across the group-V interdiffused QW for various diffusion lengths

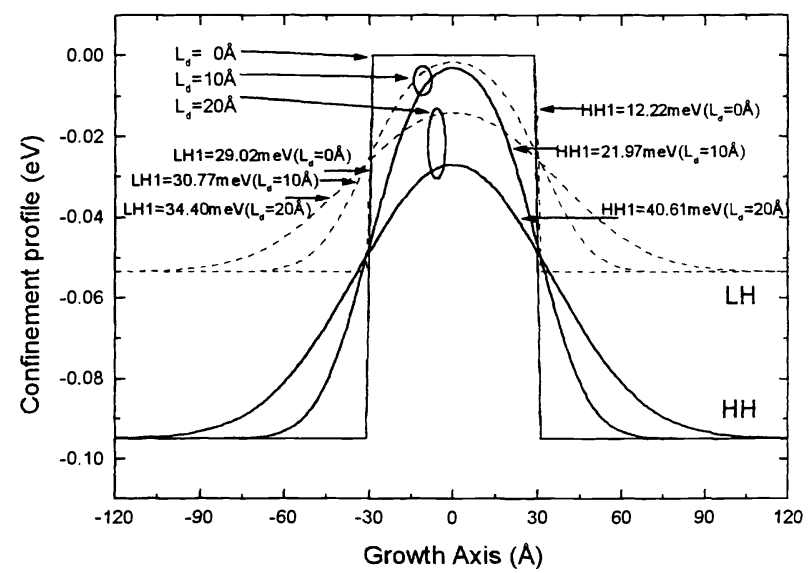

Fig. 3 The confinement potential profiles of the heavy hole (HH) and light hole (LH) subbands with different group-V diffusion lengths 

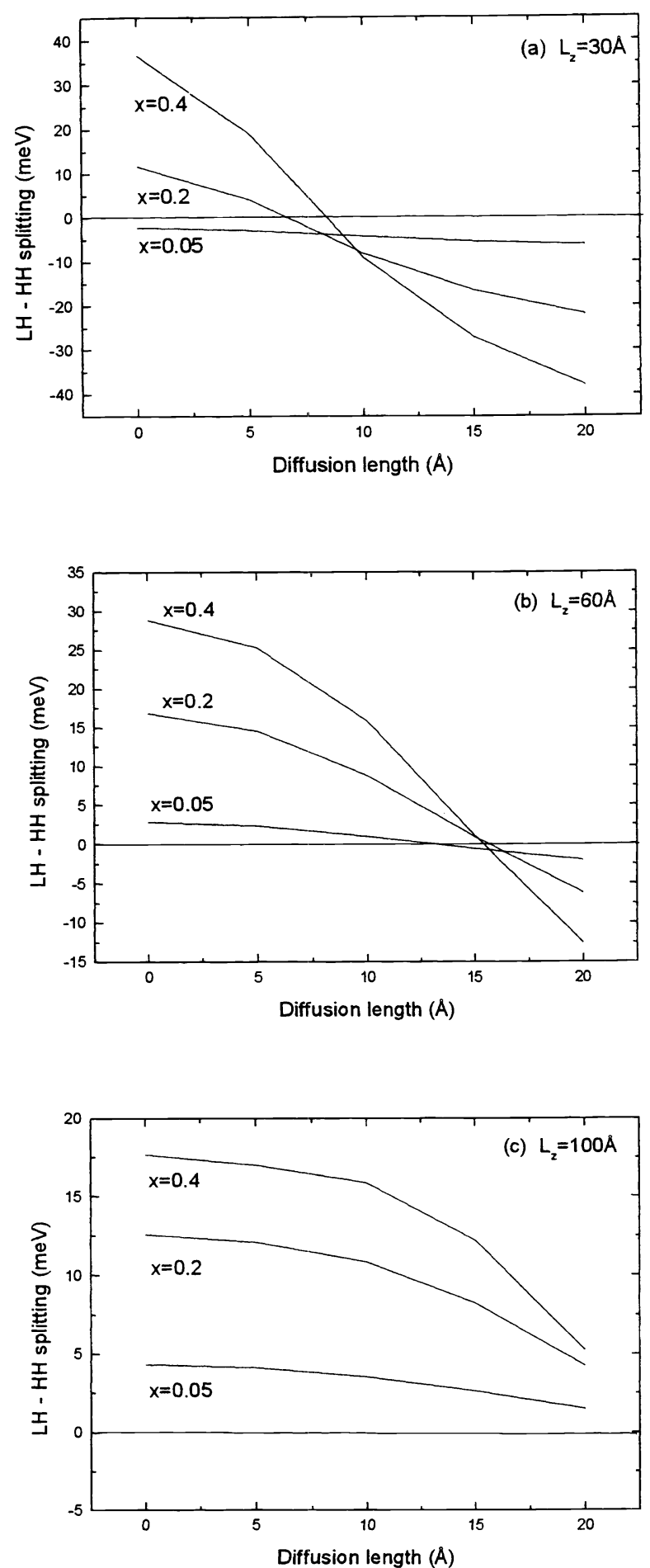

Fig. 4 The LH-HH splitting as a function of diffusion lengths for three $P$ compositions with three well width (a) $L_{z}=30 \AA$, (b) $L_{z}=60 \AA$, and (c) $\mathrm{L}_{\mathrm{z}}=100 \AA$ 
(1) variations in the depth of the potential well caused by changes in the bandgap energy; (2) the tensile strain in the barrier and well; (3) a non-constant effective well as a result of the non-square confinement profile. When $L_{d}$ is small, the $P$ concentrations near to the interface drop quickly while the concentration at the centre remains unchanged, which leads to a smaller difference of the confinement potential between heavy hole and light hole at well centre. When the diffusion length reaches $20 \AA$, the confinement potential differences will further increase. The subband-edge energy levels of heavy holes and light holes of the as-grown square QW are shifted down in the interdiffused QW structures according to the extent of interdiffusion, as shown in Table II; the zero-reference is taken to be at the top of confinement potential of the as-grown QW. The first (ground) subband-edge energy for heavy hole and light hole are shifted far away from the conduction band. The energy are shifted from 12.22 to $40.61 \mathrm{meV}$ for $\mathrm{HH}$ and from 29.02 to $34.40 \mathrm{meV}$ for $\mathrm{LH}$ with $\mathrm{L}_{2}=60 \AA$ and $\mathrm{L}_{\mathrm{d}}=0-20 \AA$. This feature is another degree of freedom to merge the $\mathrm{HH}$ and $\mathrm{LH}$ subbands together to achieve the polarization independent devices.

Table II. Subbands energy levels of heavy holes (HH) and light holes (LH).

\begin{tabular}{|l|l|l|l|l|l|}
\hline & $\mathrm{L}_{\mathrm{d}}=0 \AA$ & $\mathrm{L}_{\mathrm{d}}=5 \AA$ & $\mathrm{L}_{\mathrm{d}}=10 \AA$ & $\mathrm{L}_{\mathrm{d}}=15 \AA$ & $\mathrm{L}_{\mathrm{d}}=20 \AA$ \\
\hline HH top of confinement potential $(\mathrm{meV})$ & 0 & $2.06 \times 10^{-3}$ & -3.16 & -14.70 & -27.10 \\
\hline $\mathrm{HH} 1(\mathrm{meV})$ & -12.22 & -14.81 & -21.97 & -31.61 & -40.61 \\
\hline $\mathrm{HH} 2(\mathrm{meV})$ & -47.02 & -52.32 & -58.78 & -62.43 & -65.16 \\
\hline HH3 (meV) & -92.37 & -91.49 & -88.21 & -85.60 & -83.92 \\
\hline LH top of confinement potential $(\mathrm{meV})$ & 0 & $1.03 \times 10^{-3}$ & -1.59 & -7.52 & -14.0 \\
\hline LH1 (meV) & -29.02 & -29.38 & -30.77 & -32.57 & -34.40 \\
\hline LH2 (meV) & -60.17 & -60.02 & -59.60 & -58.90 & -58.00 \\
\hline LH3 (meV) & -63.67 & -63.73 & -63.90 & -64.11 & -64.27 \\
\hline
\end{tabular}

For the particular combination of alloy composition and QW widths, the merging of HH and LH subband can be achieved for the GaAsP/GaAs QW structures. When tensile strain is in the barrier layers with well lattice-matched to the substrate, the limitation of the $\mathrm{LH}-\mathrm{HH}$ splitting is determined by the well width and the amount of tensile strain which can modifies the confinement potential barrier height in the valence band. For the interdiffused QW, the tensile strain induced in the well layer which can also modifies the confinement potential well depth. The changes of the LH-HH splitting are also dependent on the interdiffusion. Fig. 4 shows the splitting of LH-HH as the function of diffusion length with well width of 30,60 and $100 \AA$ for the three $\mathrm{P}$ compositions. The figure shows that a larger well width for merging is required for larger diffusion lengths. When $L_{z}=100 \AA$, a diffusion length over $20 \AA$ is required to merge the LH-HH splitting. Furthermore, the small amount of $\mathrm{P}$ composition in the barrier, the LH-HH splitting is very small (between $5 \mathrm{meV}$ ).

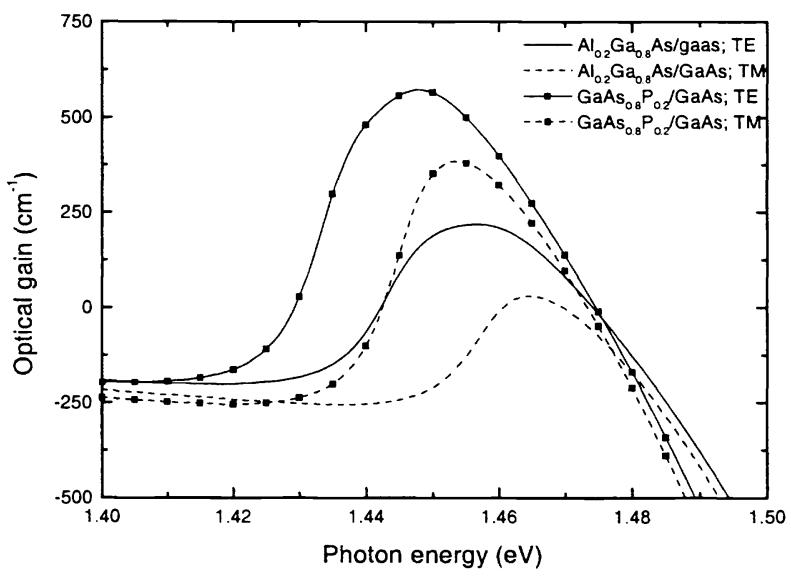

Fig.5 The TE and TM mode optical gain of the $\mathrm{Al}_{0.2} \mathrm{Ga}_{0.8} \mathrm{As} / \mathrm{GaAs}$ and $\mathrm{GaAs}_{0.8} \mathrm{P}_{0.2} / \mathrm{GaAs}$ plotted as a function of photon energy. 


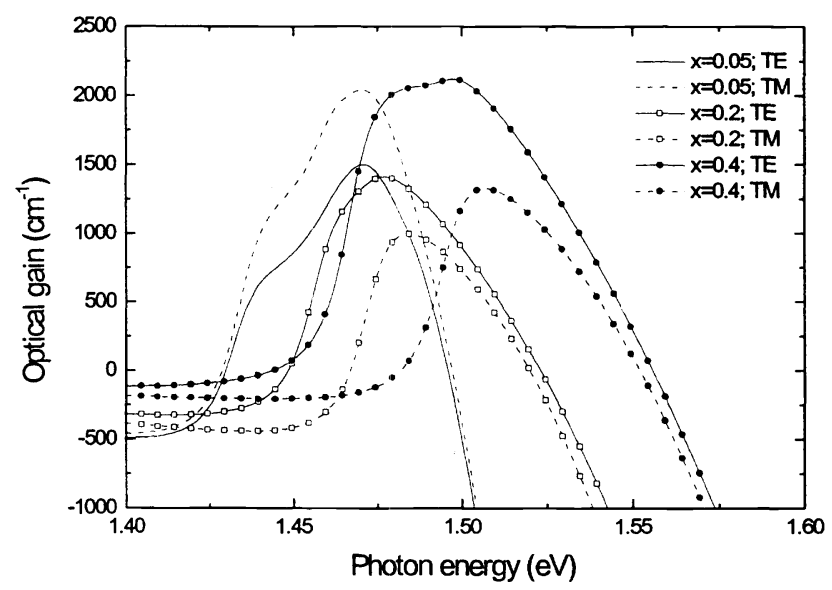

Fig.6 The TE and TM mode optical gain of the $\mathrm{GaAs}_{0.8} \mathrm{P}_{0.2} / \mathrm{GaAs}$ plotted as a function of photon energy for $\mathrm{x}=0.05,0.2$ and 0.4 .

With comparison to the typical unstrain QW structure, the optical gain results of the as-grown lattice-matched $\mathrm{Al}_{0.2} \mathrm{Ga}_{0.8} \mathrm{As} / \mathrm{GaAs}$ and tensile-strain barrier $\mathrm{GaAs}_{0.8} \mathrm{P}_{0.2} / \mathrm{GaAs} \mathrm{QW}$ with $100 \AA$ well width for $\mathrm{TE}$ and TM polarization are shown in Fig. 5. The sheet carrier density used in the calculation is $3 \times 10^{12} \mathrm{~cm}^{-2}$. The result shows that the optical gain of tensile-strain barrier QW is doublets for the unstrain QW structure. The TE and TM mode optical gain for three different $\mathrm{P}$ compositions are shown in Fig.6. For $x=0.05$, the TM mode optical gain is higher than TE mode. This is due to the small LH-HH splitting which can benefit the quantum confinement. When $\mathrm{x}=0.2$, the HH is lift above $\mathrm{LH}$, the oscillator strength for $\mathrm{HH}$ transition is dominant and for $\mathrm{LH}$ transition is reduced. The overall magnitudes for TE and TM mode optical gain are smaller than $x=0.05$. When $x=0.4$, the confinement potential well width is increased and the optical gain is also increased.

Optical gain is mainly dependent on the joint DOS, Fermi occupation factor and optical matrix element. The TE and TM mode optical gain spectra of three different $\mathrm{P}$ compositions tensile-strain barrier $\mathrm{QW}$ with various extent of interdiffusion are showed in Fig. 7(a)-(f). The magnitude of the peak gains for the TE mode and the TM mode can be seen. The carrier injection level used throughout the calculation is $3 \times 10^{12} \mathrm{~cm}^{-2}$. In the case of $\mathrm{x}=0.05$, the shift of peak gain and the change of magnitude are quite small. This is a good examples for merging the $\mathrm{LH}$ and $\mathrm{HH}$ subband which is not affect another optical properties. For $\mathrm{x}=0.2$, the TE mode optical gain is decrease as increasing the diffusion lengths while the TM mode is slightly increase and then drastically decrease to negative as $L_{d}=20 \AA$, as shown in Fig.7(c)-(d). In the case of $x=0.4$, the trends of optical gain spectra is similar to $x=0.2$ is shown Fig. 7(e)-(f). In the figure 7(f), the TM mode peak gain energy can be seen to shift towards higher energies from $\lambda=0.827 \mu \mathrm{m}(1.50 \mathrm{eV})$ of an as grown structure to $\lambda=0.79 \mu \mathrm{m}(1.57 \mathrm{eV})$ of an interdiffused QW $\left(\mathrm{L}_{\mathrm{d}}=20 \AA\right)$. In the initial stage of interdiffusion (i.e. $\mathrm{L}_{\mathrm{d}}=10 \AA$ ), an enhancement in peak gain magnitude up to $10 \%$ can be observed.

\section{CONCLUSION}

In summary, we have presented the group- $\mathrm{V}$ interdiffusion of tensile-strain barrier $G a A s_{1-x} P_{x} / G a A s$ single quantum well with well width of 30,60 , and $100 \AA$. By a suitable choice of the diffusion parameters, it is possible to cause the coincidence of energy levels of $\mathrm{HH}$ and $\mathrm{LH}$ resulting in polarization-independent operation devices. This creation of tensile strain in the well through interdiffusion results in the merging of $\mathrm{HH}$ and $\mathrm{LH}$ subbands. The interdiffusion is also an enhancement of the TM mode optical gain about $10 \%$ as compared with the as-grown wells for $0.4 \mathrm{P}$ concentration. The interdiffusion of the QW produce a blue shift of the peak gain energy, shifting from $\lambda=0.827 \mu \mathrm{m}(1.50 \mathrm{eV})$ of an as grown structure to $\lambda=0.79 \mu \mathrm{m}$ $(1.57 \mathrm{eV})$, which can be useful in bandgap tuning applications. 

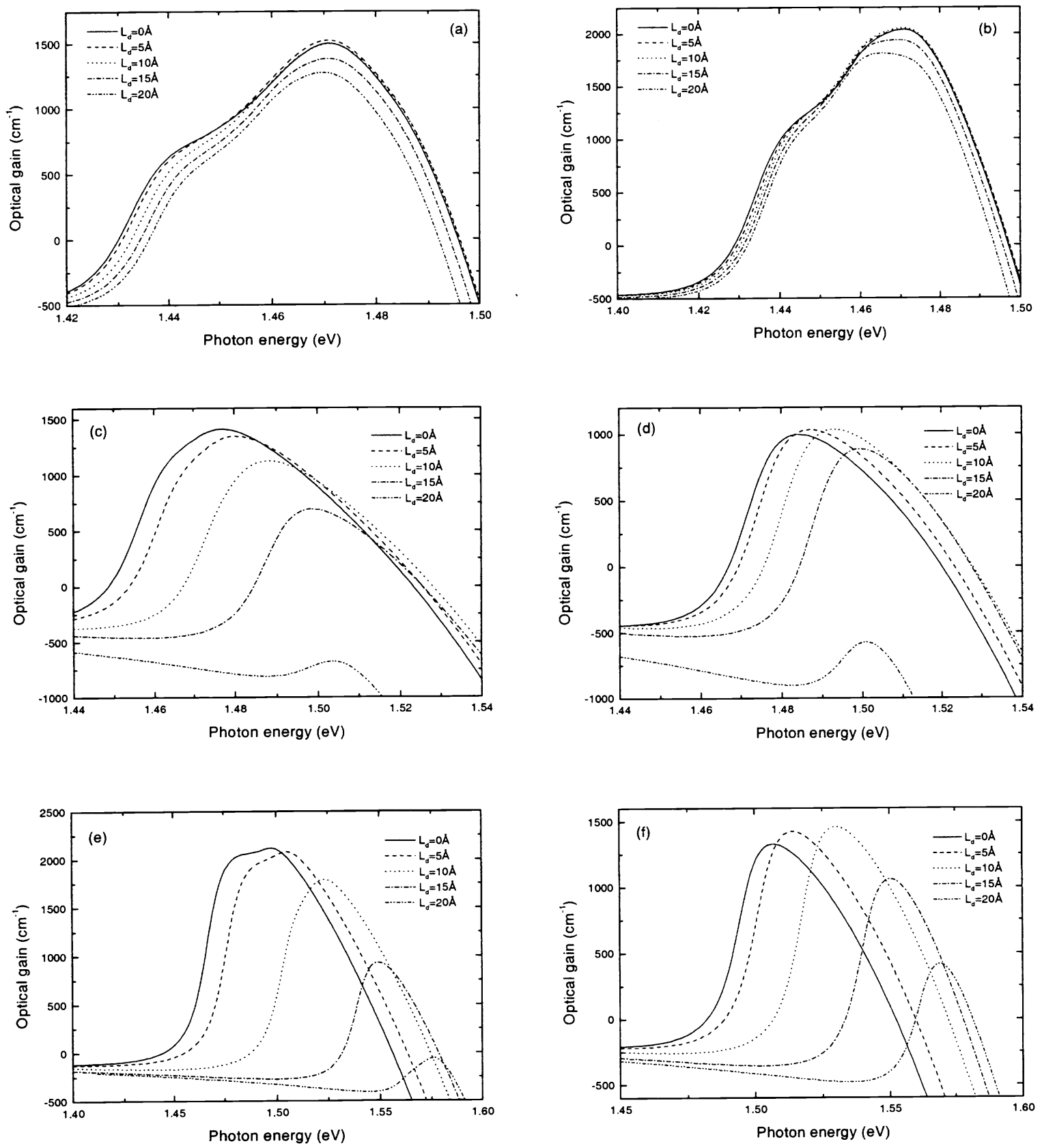

Fig.7 The optical gain of the interdiffused $\mathrm{GaAs}_{1-\mathrm{x}} \mathrm{P}_{\mathrm{x}} / \mathrm{GaAs}$ plotted as a function of photon energy for (a) $\mathrm{x}=0.05$, TE mode; (b) $\mathrm{x}=0.05$, TM mode; (c) $x=0.2$, TE mode; (d) $x=0.2$, TM mode; (e) $x=0.4$, TE mode; (f) $x=0.4$, TM mode.

\section{ACKNOWLEDGEMENT}

This paper is supported by the RGC earmarked grant of Hong Kong and the University of Hong Kong CRCG research grant. 


\section{REFERENCES}

1. R. Adams, "Band structure engineering for low-threshold high effeciency semiconductor lasers," Electron. Lett., vol. 22, pp. 249-250, 1986.

2. E. Yablonovitch and E. O. Kane, "Band structure engineering of semiconductor lasers for optical communications," IEEE. J. Lightwave Technol., vol. 6, pp. 1292-1299, 1988.

3. D. F. Welch, T. Wang, and D. R. Scifres, "Low threshold current laser emitting at 637 nm," Electron. Lett., vol. 27, pp.693-695, 1991.

4. E. Zah, R. Bhat, B. Pathak, C. Caneau, F. J. Favire, N. C. Andreadikis, D. M. Hwang, M. A. Koza, C.Y. Chen, and T. P. Lee, "Low threshold $1.5 \mu \mathrm{m}$ tensile strained single quantum well lasers," Electron. Lett., vol. 27, pp. 1414-1416, 1991.

5. P. J. A. Thijs, J. J. M. Binsma, E. W. A. Young, and W. M. A. van Gils, "High temperature operation of $\lambda=1.5 \mu \mathrm{m}$ tensile strained multiple quantum well SIPBH lasers," Electon. Lett., vol. 27, pp. 791-793, 1991.

6. L. F. Tiemeijer, P. J. A. Thijs, P. J. de Waard, J. J. Binsma, and T. V. Dongen, "Dependence of polarization, gain, linewidth enhancement factor, and $\mathrm{K}$ factor on the sign of the strain of InGaAs/InP strained-layer multi-quantum well lasers," Appl, Phys. Lett., vol. 58, pp. 2738-2740, 1991.

7. K. Magari, M. Okamoto, H. Yasaka, K. Sato, Y. Noguchi, and O. Mikami, "Polarization insensitive traveling wave type amplifer using strained multiplue quantum well structure," IEEE Photon, Technol. Lett., vol. 2, pp. 556-558, 1990.

8. Mikami, Y. Noguchi, K. Magari, and Y. Suzuki, "Polarization insensitive superluminescent diode at $1.5 \mathrm{~mm}$ with a tensile-strained barrier MQW," IEEE Photon. Technol. Lett., vol.4, pp. 703-705, 1992.

9. E. S. Koteles, "The use of strain to optimize quantum well device performance," Semi. Heterostructure for Photon. and Electron. Applications, vol. 281, pp. 141-152, 1993.

10. E.H. Li, B.L. Weiss and K.S. Chan, "Effect of Interdiffusion on the Subbands in an $\mathrm{Al}_{\mathrm{x}} \mathrm{Ga}_{1-\mathrm{x}} \mathrm{As} / \mathrm{GaAs}$ Single- QuantumWell Structure”, Phys. Rev. B, vol. 46, pp. 15181-15192, 1992.

11. W.C.Shiu, J.Micallef, I.Ng, and E.H.Li, "Effects of Different Cation and Anion Interdiffusion Rates in Disordered InGaAs/InP Single Quantum wells", Jpn. J. Appl. Phys. (Part 1), vol. 34, no.4, pp. 1778-1783, 1995.

12. J.C.P. Chang, J.M. Woodall, M.R. Melloch, I. Lahiri, D.D. Nolte, N.Y. Li, C.W. Tu, "Investigation of interface intermixing and roughening low-temperature-grown AlAs/GaAs multiple quantum wells during thermal annealing by chemical lattice imaging and x-ray diffraction”, Appl. Phys. Lett., vol. 67, pp. 3491-3493, 1995.

13. W. J. Taylor, N. Kuwata, I. Yoshida, T. Katsuyama, H. Hayashi, "Interdiffusion of GaAs/Ga1-xInxAs quantum wells," J. Appl. Phys., vol. 73, pp. 8653-8655, 1993.

14. J. Micallef, E.H. Li, B.L. Weiss, "Exciton optical absorption in disordered, strained InGaAs/GaAs single quantum wells”, Supperlattices and Microstruct., vol. 13, pp. 315-321, 1993.

15. S.S. Rao, W.P. Gillin, K.P. Homewood, "Interdiffusion of the group-III sublattice in In-Ga-As-P/In-Ga-As-P and InGa-As heterosturctures,” Phy. Rev. B, vol. 50, pp. 8071-8073, 1994.

16. K. Mukai, M. Sugawara, S. Yamazaki, "Interdiffusion process in lattice-matched $\operatorname{In}_{x} \mathrm{Ga}_{1-\mathrm{x}} \mathrm{As}_{\mathrm{y}} \mathrm{P}_{1-\mathrm{y}} / \mathrm{InP}$ and $\mathrm{GaAs} / \mathrm{Al}_{\mathrm{x}} \mathrm{Ga}_{1-}$ ${ }_{x}$ As quantum wells”, Phy. Rev. B, vol. 50, pp. 2273-2282, 1994.

17. R. Schorer, E. Friess, K. Eberl and G. Abstreiter, "Structural stability of short-period Si/Ge superlattices studied with Raman spectroscopy”, Phy. Rev. B, vol. 44, pp. 1772-1781, 1991.

18. K.P. Homewood, W.P. Gillin, R.E. Pritchard, W.S. Truscott and K.E. Singer, "Thermal processing of GaAsSb/GaAs low-dimensional strained-layer structures," Superlattic. Microstruc., vol. 7, pp. 359-361, 1990.

19. Rosenauer, T. Reisinger, E. Steinkirchner, J. Zweck and W. Gebhardt, "High resolution transmission electron microscopy determination of Cd diffusion in CdSe/ZnSe single quantum well structures," J. Crystal Growth, vol. 152, pp. 42-50, 1995.

20. D.G. Deppe and N. Holonyak, Jr., “Atom diffusion and impurity-induced layer disordering in quantum well III-V semiconductor heterostructure," J. Appl. Phys., vol. 64, pp. R93-R113, 1988.

21. Harrison, "Impurity-induced disordering in III-V multi-quantum well and superlattices," J. Mat. Sci.: Mat. in Electron., vol. 4, pp. 1-28, 1993.

22. J.H. Marsh, “Quantum well intermixing,” Semicond. Sci. Technol., vol. 8, pp. 1136-1155, 1993.

23. S. Burkner, J.D. Ralston, S. Weisser, J. Rosenzweig, E.C. Larkins, R.E. Sah and J. Fleissner "Wavelength tuning of high-speed InGaAs-GaAs-AlGaAs pseudomorphic MQW lasers via impurity-free interdiffusion," IEEE Photon. Technol. Lett., vol. 7, pp. 941-943, 1995.

24. W.C.H. Choy and E.H. Li, "The application of an interdiffused quantum well in a normally on electroabsorptive FabryPerot reflection modulator", IEEE J. Quantum Electron., vol. 33, no. 3, pp. 382-392, 1997.

25. E.S.Koteles, S. Charbonneau, P. Poole, J.J.He, M. Davies, M.Dion, G. Aers, Y. Feng, I.V. Mitchell and R.D. Goldberg, "Photonic integration using quantum well shape modification", Physics in Canada, pp.251-255, Sept/Oct 1996. 
26. D.J. Ben-Daniel and C.B. Duke, "Space-charge effects on electron tunneling“, Phys. Rev., vol. 152, pp. 683-692, 1966.

27. K. S. Chan, E. H. Li, and M. C. Y. Chan, "Optical Gain of Interdiffused InGaAs-GaAs and AlGaAs-GaAs Quantum Wells", IEEE J. Quantum Electron., vol. 34, 157-165, 1998. 